\title{
NEW LIGHT ON THE ORIGIN OF NEBULAR HEII EMISSION IN YOUNG STARBURSTS
}

\author{
DANIEL SCHAERER \\ Space Telescope Science Institute, 3700 San Martin Drive \\ Baltimore, MD 21218, USA
}

\section{Discussion}

In the Local Group few H II regions exhibit nebular He II $\lambda 4686$ emission, indicative of unusually high excitation. Approximately eight such nebulae are known, all located in low metallicity environments (IC 1613, SMC, LMC) except the Galactic ring nebula G2.4+1.4 (Esteban et al. 1992). The best studied case is the nebula S 3 surrounding the WO3 star DR 1 (Garnett et al. 1991, Kingsburgh \& Barlow 1995). Except for two cases (N 44C, N159 F; Stasińska et al. 1986, Pakull \& Angebault 1986) the nebulae are asociated with early WN (cf. Niemela et al. 1991) and WO stars.

So far approximately 50 to 60 extragalactic H II regions showing nebular He II $\lambda 4686$ are known (Campbell et al. 1986, Izotov et al. 1994, 1997a). Most of them are found in H II galaxies, BCDs and related objects from low metallicity samples used for determinations of the primordial helium abundance. The most prominent case is the low metallicity $(\mathrm{Z})$ record holder I Zw 18 . He II emission is typically on the order of $1-5 \%$ of $\mathrm{H} \beta$.

Different ionization mechanisms (photoionization by hot stars, shock excitation, photoionization by X-rays) have been put forward to explain the required hard spectrum (Garnett et al. 1991).

The first quantitative approach to address this problem are the evolutionary synthesis models of Schaerer (1996) and Schaerer \& Vacca (1997), which include well tested evolutionary tracks and recent spherically expanding non-LTE atmosphere models appropriate for WR and O stars. These models predict nebular emission due to hot WN and WC/WO stars (He II $\lambda 4686 / \mathrm{H} \beta$ up to 0.01 $0.02)$. The classification of the ionizing sources agrees well with the majority of the nebulae discussed above. As shown by Schaerer (1996) the models are also in good agreement with observations of WR galaxies showing the characteristic broad WR emission (mostly He II $\lambda 4686$ and/or C IV $\lambda 5808$ ) and/or the presence of nebular He II.

If we assume instantaneous bursts, no contamination from an underlying population and ionization bounded regions, the $\mathrm{H} \beta$ equivalent width indicates the age of the population. Comparing the observations with our recent synthesis models (Schaerer 1996, Schaerer \& Vacca 1997) we find that the vast majority of the extragalactic H II regions with nebular He II falls in the age range where WR stars are predicted to be present. This suggests that nebular emission may be related to WR stars in all cases.

The above suggestion is supported by the recent discovery of WC and WN stars in I Zw 18 (Izotov et al., 1997b, Legrand et al. 1997). Furthermore the observed nebular He II emission can be fully explained if the ionizing flux of DR 1 (Kingsburgh \& Barlow) is representative for the observed WC stars in I Zw 18.

To examine this case in more depth we have calculated new evolutionary tracks and synthesis models for $\mathrm{Z}=0.0004$ of $\mathrm{I} \mathrm{ZW} 18$. We obtain a mass limit for the formation of WR stars of $M_{\mathrm{WR}} \gtrsim$ $90 M_{\odot}$. Predictions for the WR bumps and nebular He II $\lambda 4686$ for an instantaneous burst with a Salpeter IMF extending to $150 M_{\odot}$ are shown in Figure 1. The line intensities (bottom) seem to indicate a difficulty for the models. However, the observed equivalent widths are in fair agreement, which shows that the above comparison is hampered by different spatial extensions of the gas and stars, as also recognized by Izotov et al. In particular the nebular 4686 can well be explained with a Salpeter IMF extending to a large, but not unreasonable, upper mass cut-off. Although not being 


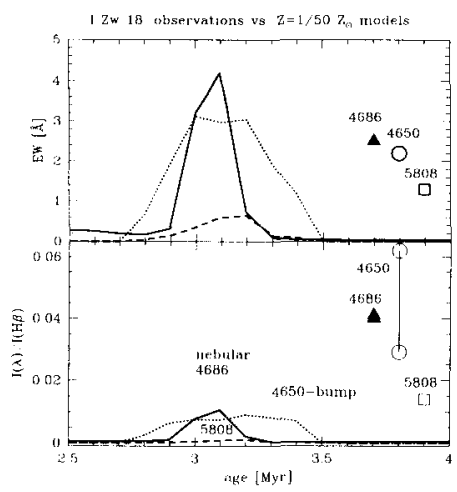

Figure 1. Predicted nebular He II $\lambda 4686$ (solid), 4650 bump (dotted), 5808 bump (dashed) for an instantaneous burst with a Salpeter IMF and $M_{\mathrm{up}}=150 M_{\odot}$ (top: equivalent widths, bottom: line intensity $I / \mathrm{H} \beta$ ). Observations from Izotov et al. (1997b) and Legrand et al. (1997) are shown on the right side. As discussed in the text $I(4650) / \mathrm{H} \beta$ shows important differences between the two observations.

a conclusive proof, the results obtained so far support the hypothesis that WR stars are responsible for nebular 4686 emission.

The observations of Izotov et al. (1997b) and Legrand et al. differ in the region of the 4650-4686 WR bump. While the former find broad emission centered at $4686 \AA$, the latter exclude any broad 4686 emission and detect a bump at $4645 \AA$, attributed to $C$ III emission from WC stars. These differences could, e.g., be due to the different slit positions. As a consequence the exact content of $\mathrm{WN}$ and $\mathrm{WC}$ stars in $\mathrm{I} \mathrm{Zw} 18$ remains uncertain.

The observed strength of the total 4650-4686 WR bump and C IV $\lambda 5808$ are well reproduced by the models. However, we predict broad $O \vee \lambda 5590$ emission with an equivalent width of approximately $1 \AA$, which does not seem to be observed. Indeed at this low $\mathrm{Z}$ the single star evolutionary models predict WN, no WC, but instead oxygen rich stars (WO). This is due to the fact that at low $\mathrm{Z}$ the He-burning core (identified with WC or WO stars according to the $\mathrm{C}, \mathrm{O}$, and He composition) is revealed at a more chemically advanced stage given the low expected mass loss. Roche lobe overflow in binary systems may have lead to the formation of WC stars. Alternatively the presence of WC stars in I Zw 18 might indicate a deficiency in the evolutionary tracks, which have so far not been tested at metallicities below the SMC. Systematic studies of the WR (WN, $\mathrm{WC}$, and possibly also WO !) and O star populations in very low metallicity $\mathrm{H}$ II galaxies are very promising in this respect. Future analysis of stellar populations in such environments should greatly improve our knowledge of massive star evolution in the early universe.

\section{Acknowledgements}

Last minute support from the IAU and a travel grant from the Swiss Society for Astronomy and Astrophysics are greatly acknowledged.

\section{References}

Campbell, A., Terlevich, R., Melnick, J. 1986, MNRAS , 223, 811

Esteban C., et al. 1992, A\&A , 259, 629

Garnett D.R., Kennicutt R.C., Chu Y.-H., Skillman E.D. 1991, ApJ , 373, 458

Kingsburgh R.L., Barlow M.J. 1995, A\&A , 295, 171

Izotov Y.I., et al. 1997b, ApJ, 487, L37

Izotov Y.I., Thuan T.X., Lipovetsky V.A. 1994, ApJ, 435, 647

Izotov Y.I., Thuan T.X., Lipovetsky V.A. 1997a, ApJS , 108, 1

Niemela V.S., et al. 1991, IAU Symp. 143, eds. K.A. van der Hucht, B. Hidayat, p. 425

Pakull M.W., Angebault, L.P. 1986, Nature, 322, 511

Legrand F. 1997, et al, astro-ph/9707279

Schaerer D. 1996, ApJ , 467, L17

Schaerer D., Vacca W.D.W. 1997, ApJS, in press

Stasińska G., Testor G., Heydari-Malayeri M. 1986, A\&A , 170, L4 